Mots. Les langages du politique

$121 \mid 2019$

Restons groupés ! La construction discursive des relations sociales

\title{
Identité verbale et modèles d'expression dans les lettres de poilus
}

Verbal identity and expression patterns in letters from the First World War Identidad verbal y modelos de expresión en las cartas de los soldados franceses

de la Primera Guerra Mundial

\section{Anne-Laure Kiviniemi}

\section{CpenEdition}

\section{Journals}

Édition électronique

URL : https://journals.openedition.org/mots/25788

DOI : $10.4000 /$ mots. 25788

ISSN : 1960-6001

Éditeur

ENS Éditions

\section{Édition imprimée}

Date de publication : 14 novembre 2019

Pagination : 109-130

ISBN : 979-10-362-0195-0

ISSN : 0243-6450

Référence électronique

Anne-Laure Kiviniemi, «Identité verbale et modèles d'expression dans les lettres de poilus », Mots. Les langages du politique [En ligne], 121 | 2019, mis en ligne le 01 janvier 2022, consulté le 22 avril 2022. URL : http://journals.openedition.org/mots/25788 ; DOI : https://doi.org/10.4000/mots.25788 


\section{Identité verbale et modèles d'expression dans les lettres de poilus}

D’aucuns se sont déjà installés pour la correspondance. Barque debout, son papier posé à plat sur un carnet dans une anfractuosité de la paroi, semble en proie à une inspiration. Il écrit, écrit, penché, le regard captivé, l'air absorbé d'un cavalier lancé au galop.

Lamuse, qui n'a pas d'imagination, passe son temps, une fois qu'il s'est assis, qu'il a posé sur la pointe matelassée de ses genoux sa pochette de papier et mouillé son crayon-encre, à relire les dernières lettres reçues, et à ne pas savoir quoi dire d'autre que ce qu'il a déjà dit, et à s'entêter à vouloir dire autre chose.

Une douceur de sentimentalité semble répandue sur le petit Eudore qui s'est recroquevillé dans une sorte de niche de terre. Il se recueille, le crayon aux doigts, les yeux sur son papier; rêveur, il regarde, il dévisage, il voit, et on voit l'autre ciel qui l'éclaire. Son regard va là-bas. Il est agrandi jusqu'à chez lui...

Le moment des lettres est celui où l'on est le plus et le mieux ce que l'on fut. (Barbusse, 1969 [1916], p. 69)

L’identité, dans la dernière phrase de cet extrait du Feu, semble conçue comme un objet à la fois :

- évolutif : il y a ce qu'on est et ce qu'on a été - le passé simple induit l'idée de perte irréversible, tandis que les superlatifs témoignent d'une tension vers ce que l'on fut sans possibilité de réinvestissement total : l'identité d'avantguerre est définitivement perdue;

et

- susceptible d'être construit discursivement, au moment des lettres.

Ces deux aspects de l'identité sont également retenus par Ruth Amossy dans son approche théorique - interactionnelle et sociodiscursive - de l'image de soi. Selon elle, toute prise de parole implique la projection d'une image de soi, comprise comme mise en scène, délibérée ou involontaire, de sa personne dans le discours. Cette image, manifestée dans le discours, se construit par le discours (ethos discursif) : "L'identité n'est pas une essence qui se traduit sur un mode plus ou moins authentique et qu'on peut exhiber ou au contraire dissimuler 
pour des besoins stratégiques [...] mais une construction verbale effectuée dans l'échange» (Amossy, 2010, p. 211). «Il ne s'agit pas de ce que le sujet est [...] mais de l'image qu'il projette dans une situation précise» (ibid., p. 27).

Or toute projection d'image de soi suppose la prise en compte de l'image qu'on croit que nos interlocuteurs ont de nous : je ne peux dire qui je suis qu'en me situant par rapport à ce que je crois être pour toi. C'est en validant, en récusant ou en infléchissant l'image que je crois que l'autre a de moi que je peux me définir. L'ethos discursif est toujours une réaction à l'ethos préalable - ma présentation de moi se fonde toujours sur l'idée que mon interlocuteur se fait d'ores et déjà de ma personne. Cet ethos préalable est compris par Ruth Amossy (ibid., p. 73) «comme l'ensemble des données dont on dispose sur le locuteur au moment de sa présentation de soi».

L'ethos préalable prédétermine donc l'ethos discursif. Construire une image de soi, c'est toujours s'engager dans un dialogue avec l'idée que les autres se font de notre personne. Et à son tour, l'ethos discursif a des conséquences sur l'ethos préalable. Le locuteur tente d'agir sur des représentations qui ne répondent plus à ses besoins identitaires.

La représentation mentale qu'élaborent les interlocuteurs fait l'objet d'une négociation qui se perpétue, puisque l'identitéest intrinsèquement inconsistante.

Les lettres des poilus apparaissent indiquées pour étudier l'ethos discursif, d'abord en raison du genre auxquelles elles appartiennent, puisque l'épistolaire - dialogue en différé et hors la vue - permet au sujet écrivant de négocier son ethos préalable sans subir les aléas de la conversation en face-à-face. Ensuite, le contexte historique a des incidences sur l'image que les correspondants veulent donner d'eux-mêmes et sur l'image qu'ils croient que les autres ont d'eux.

L'objectif sera de déterminer comment les scripteurs usent des pronoms personnels pour manifester leur présence et leurs appartenances. Car il faut aussi considérer le fait que l'ethos, qu'il soit discursif ou préalable, se compose de l'identité individuelle du locuteur (ethos personnel) et de son identité sociale liée à ses appartenances de groupe (ethos collectif). L'ethos personnel est l'image que le locuteur projette de sa personne, tandis que l'ethos collectif est l'image attachée à un ou plusieurs groupes auxquels le locuteur appartient et qui fondent son identité.

D’autre part, toute présentation de soi se nourrit de représentations collectives:

Qu'elle soit individuelle ou collective, la construction d'une image de soi est toujours tributaire d'un imaginaire social. C'est [...] en fonction de normes partagées, que je construis une identité à l'intention de mes partenaires. (Amossy, 2010, p. 44)

L'identité, modulée au fil des interactions auxquelles le locuteur prend part, est instable, multiple et « étroitement liée à la question de l'efficacité verbale » (ibid., p. 212). L'identité se forme dans l'usage de la langue. 


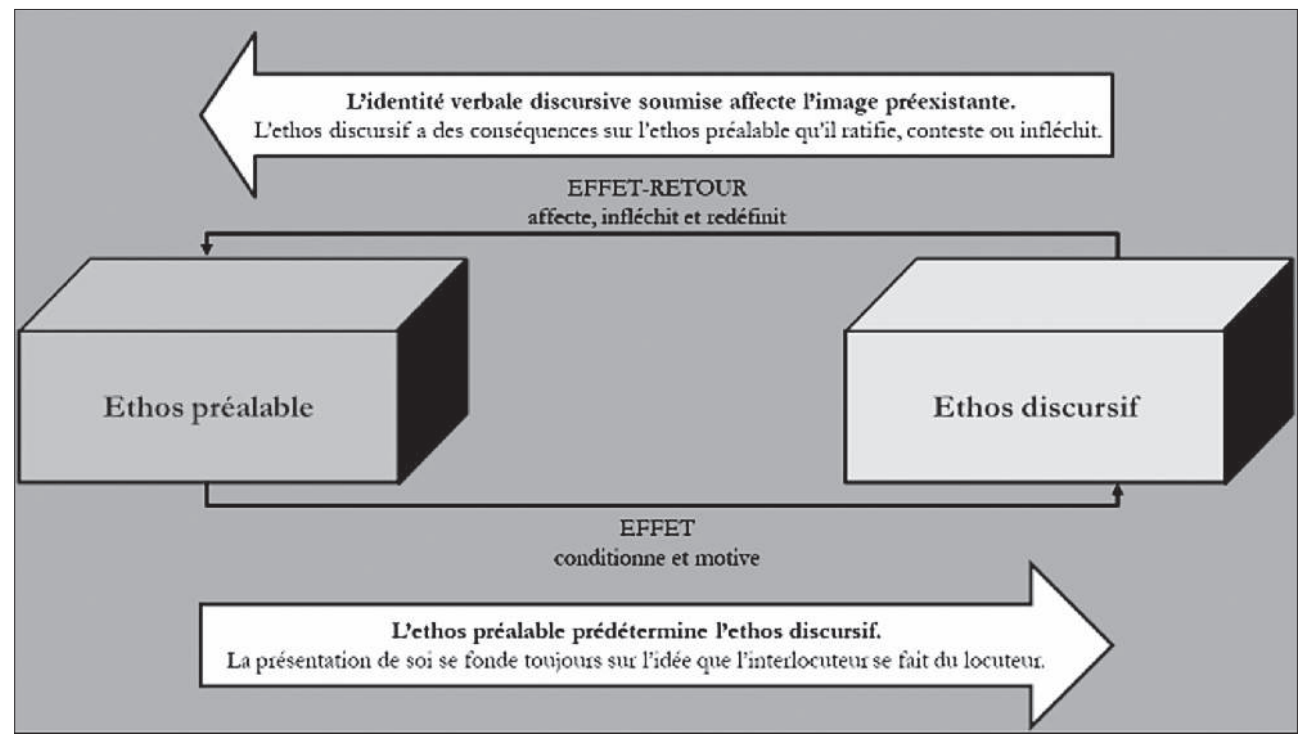

Figure 1. Ethos préalable et ethos discursif

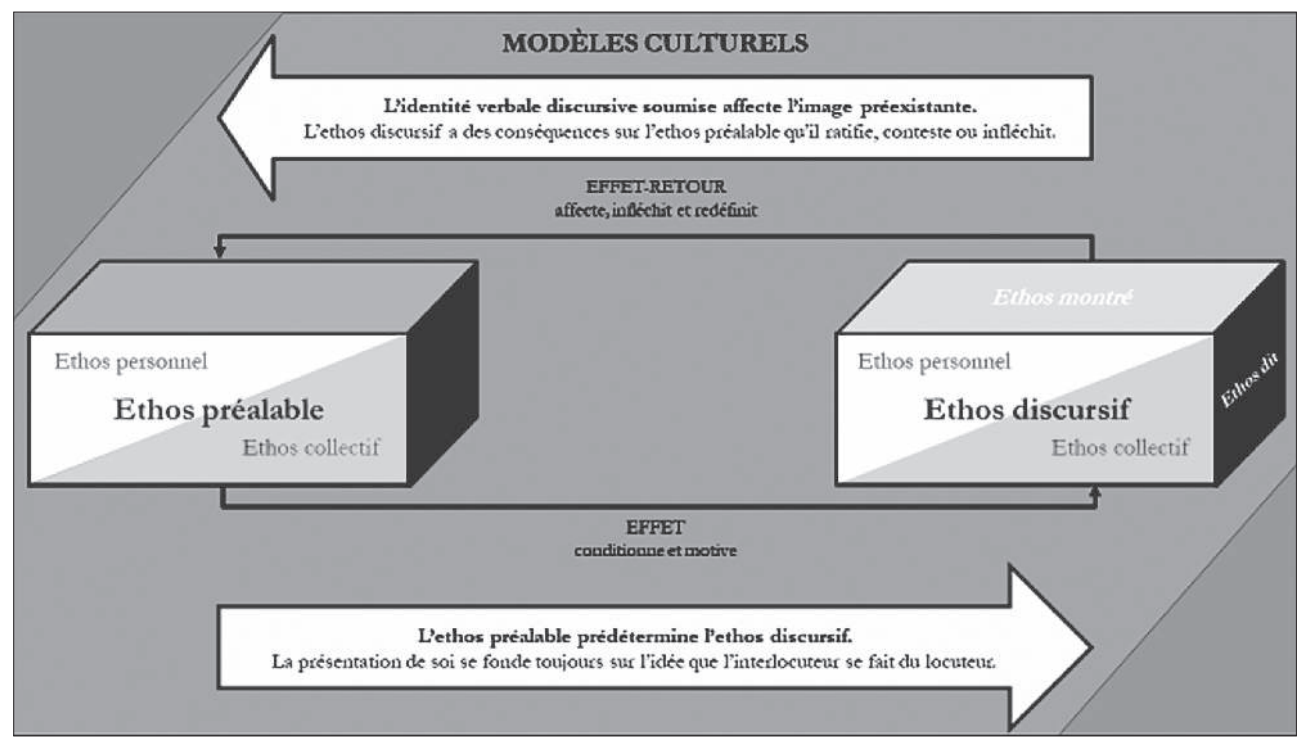

Figure 2. Construction de l’identité verbale 
L'image de soi peut découler du dit : ce que le locuteur énonce explicitement sur lui-même en se prenant comme thème de son propre discours. En même temps, elle est toujours un résultat du dire : le locuteur se dévoile dans les modalités de sa parole, même lorsqu'il ne se réfère pas à lui-même. C'est ce que Maingueneau a appelé [...] ethos dit et ethos montré. (Amossy, 2010, p. 113)

L'ethos dit est ce que le locuteur dit sur lui-même et l'ethos montré, ce que montre sa manière d'énoncer.

Pour résumer, l'ethos discursif, personnel et collectif, tributaire de modèles culturels, se forme à partir d'un ethos dit et d'un ethos montré qui s'exercent à négocier un ethos préalable, en le ratifiant, le contestant ou l'infléchissant dans le sens - délibéré ou irréfléchi - du locuteur. C'est par les modalités du dire des poilus - leurs usages des pronoms personnels, plus que par ce qu'ils disent d'eux-mêmes ${ }^{1}$ - que nous voulons interroger l'identité verbale qu'ils construisent dans leurs lettres.

Comment les scripteurs, dans leurs manières d'énoncer (ethos montré), reprennent et réactivent l'image qu'on peut se faire d'eux (ethos préalable), la modulent ou essayent de la modifier en profondeur? Les scripteurs sont tous des produits de l'école de la Troisième République, qui avait notamment pour rôle de diffuser dans l'ensemble de la société française une langue commune, le français national. Il s'agissait de «communiquer les connaissances des règles de la langue nationale à tous les enfants de la nation, sous la forme des Éléments de la Grammaire » (Balibar, 1999, p. 258). Ce français civique élémenté (simplifié de manière à donner des notions élémentaires) était inculqué à l'école primaire, tandis que, dans le secondaire, on continuait à enseigner un français humaniste 2 (la grammaire française raisonnée héritière de Port-Royal avec la comparaison systématique des langues mortes et des langues vivantes et l'étude d'auteurs littéraires, modèles d'écriture et de rhétorique). Ainsi «la langue de la Leçon de choses et la langue de la dissertation d’idées étaient instituées dans des établissements séparés » (Balibar, 1985, p. 406), la première à «l'école du peuple», de 6 à 13 ans, la seconde à «l'école des notables»3, de 8 à 18 ans (Prost, 1968).

Aux classes dirigeantes, l'école secondaire avec la culture classique ou scientifique dont elles ont besoin et qu'elles peuvent payer; pour le peuple, les rudiments suffisent, pourvu qu'ils soient imprégnés de moralité et inculquent l'obéissance. (Prost, 1968, p. 10)

L'identité verbale construite par les épistoliers est-elle en prise directe sur les modèles d'écriture avec lesquels ils ont été familiarisés?

1. Sur cet aspect, voir Vicari, 2012, Housiel, 2014 ou Steuckart éd., 2015.

2. Les termes sont de Renée Balibar (1985, p. 251). Le français civique est celui qui permet de développer les échanges entre tous les citoyens ; le français humaniste est celui qui permet de développer les échanges entre privilégiés de la République des lettres.

3. Les termes sont d'Antoine Prost (1968). 


\section{La référence personnelle dans les lettres de poilus : vue d'ensemble}

L'analyse des pratiques de présentation de soi se fera par l'examen des pronoms personnels dans des correspondances de guerre. La présence du scripteur dans son texte se manifeste en effet d'abord par l'utilisation des pronoms personnels - repères de subjectivité traduisant l'appropriation de son propre discours par le sujet parlant (Benveniste, 1966). La référence aux actants de la communication informe sur l'image de soi : "l'analyse des pratiques de présentation de soi commence nécessairement parl'examen des personnes grammaticales [...] [qui] soulèvent des questions de fond sur la nature et les fonctions de l'ethos» (Amossy, 2010, p. 103).

Un relevé des indices personnels (tableau 1) a été effectué dans les échanges épistoliers de quatre soldats de parcours scolaires différant par leur longueur et par leur contenu. Seront étudiés les écrits de Baptiste Lapouge, peu-lettré de l'école publique, ceux d'André Boulo et d'Henri Rivière qui ont tous deux obtenu le certificat d'études, le premier en effectuant sa scolarité dans une école catholique, le second dans une école publique, et ceux d'André Fugier, bachelier de l'école privée4. Les fonds Fugier, Rivière et Boulo sont conservés au centre de documentation de l'Historial de la Grande Guerre, à Péronne. Ils ont été choisis parmi les correspondances en français selon les critères suivants :

- quantité de lettres constituant l'échange : les échanges épistolaires contenant moins d'une cinquantaine de lettres ont été éliminés;

- existence d'informations sur l'auteur de l'échange : les descriptions fournies par les donateurs des documents authentiques ont orienté le choix, car il fallait sélectionner des fonds écrits par des scripteurs d'habileté différente, donc de niveaux d'instruction et de parcours scolaires divers;

- diversité du fonds : les fonds contenant des documents complémentaires (photographies, carnet de guerre, généalogie, etc.) ont été privilégiés.

Des recherches supplémentaires ont toutefois été nécessaires pour l'obtention de l'échange épistolaire conséquent d'un peu-lettré. C'est sur le site internet du Crid 14-185 qu'a été trouvée mention du fonds Lapouge se trouvant au Centre d’études Edmond Michelet à Brive. Les archives nous sont parvenues sous forme de cédérom numérisé.

4. B. Lapouge (1885-1973) est un paysan corrézien qui écrit à sa femme tout au long du conflit. A. Boulo (1897-1965), après une formation de mécanicien, part à la guerre en février 1917 et correspond avec ses parents jusqu'à la fin de son service militaire en août 1919. H. Rivière (18821916) est employé de bureau dans une usine de tissage. Il écrit à son frère entre novembre 1914 et septembre 1916, date de son décès sur le front. A. Fugier (1896-1966) se destine à la carrière militaire. Sa correspondance commence en 1915 et s'arrête en 1917 alors qu'il est grièvement blessé lors d'une attaque (amputation de la jambe droite).

5. http://www.crid1418.org/ (consulté le 20/05/2019). 
Le fonds Lapouge contient 99 documents, dont 63 lettres ou cartes écrites par Baptiste entre 1914 et 1918. Le fonds Boulo contient 55 lettres pour la période de la guerre (entre le 21 juin 1917 et le 8 novembre 1918) et 31 pour la période d'occupation en Allemagne (jusqu'au 16 août 1919). Le fonds Rivière comprend 326 lettres et cartes rédigées entre novembre 1914 et septembre 1916. Le fonds Fugier est composé de 254 lettres écrites entre octobre 1915 et janvier 1918, d'un journal de guerre, de photographies et de dessins du front et d'un carnet de campagne réalisé après la guerre. Pour faciliter l'analyse comparative, 50 lettres par soldat ont été choisies en privilégiant les lettres du front. Les pièces d'archives des fonds susmentionnés, qu'elles soient numérisées (fonds Lapouge) ou photographiées (fonds Boulo, Rivière, Fugier), ont été transcrites au plus près des textes originaux ${ }^{6}$.

Avec l'usage de la première personne du singulier sera appréhendée la construction identitaire individuelle du locuteur (partie 2); avec l'usage de la première personne du pluriel, l’identité sociale liée aux appartenances de groupe (partie 3). La terminologie mobilisée dans le tableau 1 affiche les présupposés théoriques retenus : nous suivons Benveniste (1966, p. 228) sur la distinction personne subjective / personne non subjective, et nous conformons à Kerbrat-Orrechioni (1980, p.41), Guespin (1985, p. 50) et Labbé (1998, p. 4) sur la distinction nous inclusif / nous exclusif.

Dans les premières personnes, il y a à la fois une personne impliquée et un discours sur cette personne. Je désigne celui qui parle et implique en même temps un énoncé sur le compte de je : disant je, je ne puis pas ne pas parler de moi. À la $2^{\mathrm{e}}$ personne, tu est nécessairement désigné par je et ne peut être pensé hors d'une situation posée à partir de $j e$; et en même temps, je énonce quelque chose comme prédicat de tu. [...] on pourra définir le tu comme la personne non subjective, en face de la personne subjective que je représente. (Benveniste, 1966, p. 228)

Dans les lettres de soldats, je est celui qui écrit la lettre; le tu et le vous sont les allocutaires, en général aussi destinataires du discours. Vous peut cependant désigner quelquefois un collectif (vous déictique et cotextuel), par exemple la communauté géographique d'origine du scripteur :

(1) nous avons toujour ce mauvez temp il doi bien faire de même au pays ce qui ne vous avance pas beaucoup pour vos traveaux.7 (Fonds Lapouge, lettre du 04/06/1915)

plus rarement un datif éthique (vous exclusif) :

6. Les transcriptions ont été éditées dans leur intégralité dans la seconde partie de ma thèse de doctorat. Pour les lire, consulter : http://urn.fi/URN:ISBN:978-952-03-0450-8 (consulté le 20/05/2019).

7. L'orthographe et la présentation des textes du corpus ont été scrupuleusement respectées pour éviter autant que possible qu'interprétation et transcription ne se confondent. 
(2) L'on touche de nouvelles capotes gris clair qui vous font ressembler aux Bôches. (Fonds Rivière, lettre du 27/02/1915)

Vous est aussi sporadiquement utilisé dans le discours cité et dans des expressions idiomatiques.

Le nous renvoie à un ensemble de personnes auxquelles appartient le scripteur. Selon la composition de cet ensemble peuvent être distingués :

- nous $_{1}$, nous inclusif : je + destinataire(s)

(3) je voudrais comme autrefois revivre auppres de toi nous ettion et nous serion encore si heureux de pouvoir nous partager nos soufrance et nos joie par des inombrables Baisers (Fonds Lapouge, lettre du 17/02/1915)

- nous $_{2}$, nous inclusiflarge : je + communauté non limitée

(4) Mon epouse bien aimer Nous voici dimanche (Fonds Lapouge, lettre du 23/01/1916)

- nous $_{3}$, nous exclusif: je + les soldats

(5) Nous avons été relevés ce matin (Fonds Fugier, lettre du 18/10/1915)

Pour une complète prise en compte des réalisations des différentes personnes ont été aussi recensés, à la manière d'Anna Jaubert (1987), formes toniques, compléments, adjectifs et pronoms possessifs. Le on = nous n'est pas reproduit dans le tableau pour cause d'interprétation délicate : la valeur de base du on, «indéfinie, n'est jamais totalement supprimée » (Rabatel, 2001, p. 32). Une analyse qualitative semble dans ce cas plus appropriée. 


\begin{tabular}{|c|c|c|c|c|c|}
\hline & & $\mathrm{AF}$ & AB & $\mathrm{HR}$ & $B L^{a}$ \\
\hline \multicolumn{2}{|l|}{ Nombre de mots } & 25498 & 30489 & 20853 & 16019 \\
\hline \multicolumn{2}{|c|}{ La personne subjective et ses autres réalisations } & 934 & 1594 & 1236 & 1356 \\
\hline personne subjective & je & 515 & 985 & 714 & 692 \\
\hline \multirow[t]{7}{*}{ formes toniques/compléments } & & 256 & 438 & 276 & 229 \\
\hline & moi & 44 & 111 & 35 & 35 \\
\hline & moi-même & 0 & 2 & 5 & 1 \\
\hline & moi aussi & 1 & 2 & 4 & 1 \\
\hline & moi non plus & 0 & 1 & 0 & 0 \\
\hline & m' & 106 & 145 & 93 & 67 \\
\hline & me & 105 & 177 & 139 & 125 \\
\hline \multicolumn{2}{|c|}{$\begin{array}{l}\text { toutes fonctions de la } 1^{\text {re }} \text { personne confondues } \\
\text { (personne subjective + formes toniques/ } \\
\text { compléments) }\end{array}$} & 771 & 1423 & 990 & 919 \\
\hline \multirow[t]{8}{*}{ adjectifs et pronoms possessifs } & & 163 & 171 & 246 & 437 \\
\hline & mon & 46 & 49 & 54 & 85 \\
\hline & $\mathrm{ma}$ & 51 & 93 & 102 & 287 \\
\hline & mes & 62 & 29 & 73 & 62 \\
\hline & mien & 0 & 0 & 1 & 0 \\
\hline & miens & 0 & 0 & 6 & 2 \\
\hline & mienne & 3 & 0 & 10 & 0 \\
\hline & miennes & 1 & 0 & 0 & 1 \\
\hline \multicolumn{2}{|c|}{ La personne non subjective et ses autres réalisations } & 13 & 185 & 584 & 791 \\
\hline personne non subjective & tu & 4 & 78 & 139 & 157 \\
\hline \multirow[t]{6}{*}{ formes toniques / compléments } & & 7 & 54 & 293 & 450 \\
\hline & toi & 1 & 9 & 36 & 69 \\
\hline & toi-même & 0 & 0 & 0 & 1 \\
\hline & toi aussi & 0 & 0 & 3 & 1 \\
\hline & t' & 2 & 19 & 114 & 210 \\
\hline & te & 4 & 26 & 140 & 169 \\
\hline \multicolumn{2}{|c|}{$\begin{array}{l}\text { toutes fonctions de la } 2^{\mathrm{e}} \text { personne confondues } \\
\text { (personne non subjective + formes toniques/ } \\
\text { compléments) }\end{array}$} & 11 & 132 & 432 & 432 \\
\hline \multirow[t]{8}{*}{ adjectifs et pronoms possessifs } & & 2 & 53 & 152 & 184 \\
\hline & ton & 0 & 25 & 76 & 69 \\
\hline & ta & 1 & 24 & 38 & 34 \\
\hline & tes & 1 & 3 & 35 & 77 \\
\hline & tien & 0 & 0 & 0 & 0 \\
\hline & tiens & 0 & 0 & 1 & 2 \\
\hline & tienne & 0 & 1 & 2 & 0 \\
\hline & tiennes & 0 & 0 & 0 & 2 \\
\hline
\end{tabular}


Identité verbale et modèles d'expression dans les lettres de poilus

\begin{tabular}{|c|c|c|c|c|c|}
\hline & & $\mathrm{AF}$ & $A B$ & HR & $B L^{a}$ \\
\hline \multicolumn{2}{|l|}{ Vous et ses autres réalisations } & 244 & 206 & 57 & 16 \\
\hline \multicolumn{2}{|l|}{ Vous } & 224 & 170 & 41 & 13 \\
\hline \multirow{3}{*}{$\begin{array}{l}\text { déictique pur (un allocutaire; vous } \\
\text { de politesse) }\end{array}$} & vous & 0 & 0 & 24 & 0 \\
\hline & votre & 0 & 0 & 11 & 0 \\
\hline & vos & 0 & 0 & 3 & 0 \\
\hline \multirow[t]{3}{*}{ déictique pur (allocutaires) } & vous & 220 & 123 & 8 & 7 \\
\hline & votre & 17 & 33 & 1 & 1 \\
\hline & vos & 3 & 1 & 0 & 0 \\
\hline \multirow{3}{*}{$\begin{array}{l}\text { déictique et cotextuel } \\
\text { (allocutaire(s) + non-allocutaire(s)) }\end{array}$} & vous & 3 & 36 & 1 & 6 \\
\hline & votre & 0 & 0 & 1 & 0 \\
\hline & vos & 0 & 2 & 0 & 2 \\
\hline $\begin{array}{l}\text { datif éthique de prise à témoin de } \\
\text { l'interlocuteur }\end{array}$ & vous & 1 & 5 & 8 & 0 \\
\hline discours cité & vous & 0 & 4 & 0 & 0 \\
\hline expressions idiomatiques & vous & 0 & 2 & 0 & 0 \\
\hline \multicolumn{2}{|c|}{$\begin{array}{l}\text { Manifestations du/des destinataires } \\
\text { (personne non subjective + vous déictique pur) }\end{array}$} & 253 & 342 & 631 & 799 \\
\hline \multicolumn{2}{|l|}{ Nous et ses autres réalisations } & 576 & 334 & 183 & 304 \\
\hline \multicolumn{2}{|l|}{ Nous } & 485 & 287 & 114 & 244 \\
\hline \multirow{5}{*}{$\begin{array}{l}\text { nous inclusif (je + destinataire(s)) } \\
\text { autres réalisations }\end{array}$} & nous & 1 & 4 & 13 & 27 \\
\hline & nous-mêmes & 0 & 0 & 0 & 0 \\
\hline & notre & 0 & 1 & 18 & 5 \\
\hline & nos & 1 & 0 & 20 & 5 \\
\hline & nôtre & 0 & 0 & 0 & 0 \\
\hline total & & 2 & 4 & 51 & 37 \\
\hline \multirow{3}{*}{$\begin{array}{l}\text { nous inclusif large } \\
\text { (je + communauté non limitée) } \\
\text { autres réalisations }\end{array}$} & nous & 0 & 7 & 7 & 4 \\
\hline & notre & 0 & 1 & 0 & 0 \\
\hline & nos & 0 & 0 & 4 & 0 \\
\hline total & & 0 & 9 & 11 & 4 \\
\hline \multirow{5}{*}{$\begin{array}{l}\text { nous exclusif (je + les soldats) } \\
\text { autres réalisations }\end{array}$} & nous & 484 & 276 & 94 & 212 \\
\hline & nous-mêmes & 3 & 0 & 0 & 0 \\
\hline & notre & 45 & 30 & 14 & 21 \\
\hline & nos & 40 & 13 & 13 & 29 \\
\hline & nôtre & 2 & 2 & 0 & 0 \\
\hline total & & 574 & 321 & 121 & 262 \\
\hline
\end{tabular}

a. La segmentation des mots (apostrophes et tirets) a été rétablie dans le texte de B. Lapouge pour avoir des données comparables à celles des autres soldats.

Tableau 1. Les indices personnels dans les correspondances Fugier, Boulo, Rivière et Lapouge 
Passons donc maintenant en revue les usages singuliers des scripteurs : comment les pronoms sont-ils utilisés et comment réfèrent-ils? Des différences sont-elles perceptibles dans la correspondance des quatre soldats?

\begin{tabular}{|c|c|c|c|c|}
\hline & $\mathrm{AF}$ & $A B$ & HR & BL \\
\hline La personne subjective et ses autres réalisations & $3,7 \%$ & $5,2 \%$ & $5,9 \%$ & $8,5 \%$ \\
\hline Manifestations du/des destinataires & $1 \%$ & $1,1 \%$ & $3 \%$ & $5 \%$ \\
\hline Nous et ses autres réalisations & $2,3 \%$ & $1,1 \%$ & $0,9 \%$ & $1,9 \%$ \\
\hline $\begin{array}{l}\text { dont nous exclusif (je }+ \text { les soldats) } \\
\text { et ses autres réalisations }\end{array}$ & $2,3 \%$ & $1,1 \%$ & $0,6 \%$ & $1,6 \%$ \\
\hline
\end{tabular}

Tableau 2. La référence personnelle chez A. Fugier, A. Boulo, H. Rivière et B. Lapouge Statistiques élaborées à partir du tableau 1 (pourcentages calculés sur le nombre total de mots de chacun des sous-corpus)

La lecture du tableau 2 apporte deux enseignements : 1) en ce qui concerne l'identité individuelle, le recours à la personne subjective recule avec le degré d'instruction du scripteur; 2) en ce qui concerne la construction sociale, les scripteurs se catégorisent avant tout comme soldats.

\section{Image de soi et construction identitaire individuelle : comment l’épistolier se présente-t-il?}

Le décompte des marques personnelles de ce corpus restreint met en valeur une corrélation entre le degré d'instruction des scripteurs et l'emploi de la première personne. L'actualisation du sujet discoureur à travers la personne subjective dans ces écrits semble être en raison inverse de la scolarisation du scripteur. Dans les lettres d'A. Fugier, on recense 934 explicitations de l'énonciateur dans un corpus de 25498 mots, soit 3,7\%. Chez B. Lapouge, ce sont 1356 manifestations de la première personne sur 16019 mots, soit 8,5\%. La différence est de taille surtout si l'on prend en considération le fait que B. Lapouge, le peulettré corrézien, pratique fréquemment l'ellipse du sujet.

Il s'agit ici d'un emprunt structurel de l'occitan vers le français. L'occitan a conservé des désinences verbales exprimant par elles-mêmes la personne, ce qui rend redondant l'emploi des pronoms sujet. Baptiste transpose cette manière de faire en français, lorsque désinence verbale et contexte permettent de ne pas avoir de doute sur la personne. (Klippi, Kiviniemi, 2015, p. 181)

Comment interpréter ces différences dans l'implication subjective des scripteurs? L'effacement de la personne, plus marqué dans les écrits du bachelier que dans ceux du peu-lettré, pourrait manifester un discours plus narratif que conversationnel : «le Sujet discoureur s'actualise beaucoup plus modestement dans la fiction » (Jaubert, 1990, p.12). Cette hypothèse est étayée par 


\section{Langage de proximité}

(1) communication privée

(2) interlocuteur intime

(3) émotionnalité forte

(4) ancrage actionnel et situationnel

(5) ancrage référentiel dans la situation

(6) coprésence spatio-temporelle

(7) coopération communicative intense

(8) dialogue

(9) communication spontanée

(10) liberté thématique

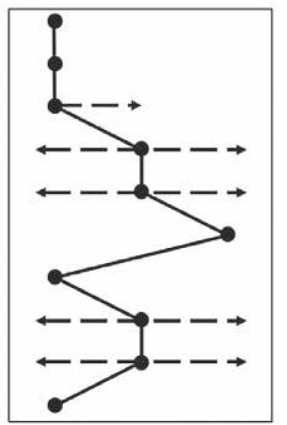

Langage de distance

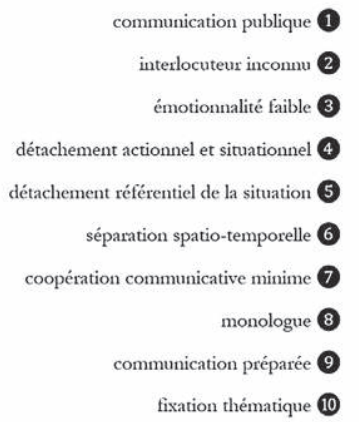

$\longrightarrow$ lettre intime

variation selon les lettres

Figure 3. Relief conceptionnel des lettres intimes

Application du modèle de P. Koch et W. Oesterreicher (2001, p. 586)

reproduit selon le modèle de S. Bibauw (2007, p.51). (Klippi, Kiviniemi, 2015)

la prise en compte des fréquences d'apparence des tu/vous destinataires et des datifs éthiques de prise à témoin de l'interlocuteur. Au vu des résultats du tableau (et cette interprétation proposée sur la base des lettres étudiées devra, pourvalidation, être confrontée à des corpus plus étendus en quantité et sur la durée), les moins scolarisés auraient une approche plus dialogale de la communication épistolaire, se conformant ainsi aux instructions des manuels primaires : «La lettre est une conversation écrite entre personnes qui ne peuvent facilement se voir à cause de l'éloignement» (Viala, 1896, p. 257); tandis que la part de narration augmenterait avec la durée du cursus du scripteur et sa familiarité avec le littéraire. «Dans l'enseignement secondaire, la composition repose sur des bases solides : l'apprentissage du cours de rhétorique accompagné de la lecture, de la mémorisation et de l'imitation des auteurs » (Chervel, 2006, p.698).

L'épistolaire, en tant que genre oscillant entre langage de proximité et langage de distance (figure 3$)^{8}$, permet aux scripteurs de choisir, dans leurs lettres, entre les deux pôles que sont le mimétisme d'une conversation et la narration autobiographique.

La lettre personnelle [...] est censée supporter la moitié d'un dialogue entre deux êtres, mais un dialogue en différé et hors la vue [...] le scripteur, libéré de la présence physique de l'Autre [...] peut à loisir projeter un Destinataire idéal, lecteur sur mesure de ses complaisances introspectives, narratives parfois. (Jaubert, 1990, p.11)

8. Ce schéma est applicable aux lettres intimes en général. 
C'est le cas d'A. Fugier, qui se construit une image de narrateur par la littérarisation de son discours.

(6) Le jour, je fais le tour de mes domaines, casque en tête et canne à la main, tel Sancho Pança visitant son île. (Fonds Fugier, lettre du 18/10/1915)

(7) Chers parents - On liquide, on s'en va - On fait le sac, on boucle la cantine et de nouveau, nous voici partis dans le monde. (Fonds Fugier, lettre du 30/12/1915)

Les moins lettrés aussi cherchent sporadiquement à se détacher de l'image d'épistolier pour endosser celle d'écrivain, comme ces passages exaltés le prouvent :

(8) je t assure mas cherie que jen est assez de cette vie de suplice a la Fleur de nottre âge au moment qu on allez ettre si heureux c est bien dur pour moi de nous avoir separer. (Fonds Lapouge, lettre du 28/04/1915)

(9) par ce temps l'on est plus tranquille qu'au cantonnement. L'on examine à loisir les aéros qui sillonnent l'azur mais il faut se garer quand même car les canons les canardent à sckrapnels que veux tu, et les éclats peuvent vous atteindre. L'on se sent revivre car les effluves et les senteurs commencent à emplir notre être. Je voudrais être poète pour bien m'exprimer. (Fonds Rivière, lettre du 05/02/1915)

Mais l'interaction avec le destinataire pénètre jusque dans ces passages narratifs, comme en témoigne par exemple l'appellatif mas cherie.

La lecture attentive des lettres du corpus montre que derrière les différences quantitatives dans la part de narration d'un échange épistolier à l'autre se trouve un fonctionnement similaire :

[...] il faut reconnaître dans le texte des secteurs significatifs [...], distinguer ceux qui répondent aux critères du dialogue, structurés par «la relation discursive au partenaire » [...] et ceux qui offrent bien davantage les caractères de la narration autobiographique. (Jaubert, 1987, p. 21)

Il faut faire ainsi la part des choses entre le je qui écrit - celui qui construit le monde épistolaire (niveau performatif) - et le je qui raconte - celui qui rapporte des nouvelles (niveau narratif). Les lettres, conformément aux conventions épistolaires9, commencent généralement par une introduction dialogale avec l’intervention d'un je énonciateur qui amène le je autobiographique :

[...] tout se passe comme si écrire une lettre, ce n'était pas seulement aller à la rencontre de l'autre, c'était aussi, sous ce couvert, aller à la rencontre de soi-même; par ce trait [...] le genre de la lettre accède à son statut littéraire. (Jaubert, 1987, p. 24)

9. "Traditionally, the format of the ars dictaminis followed a standard five-part structure: the salutatio, a formulaic greeting, the captatio benevolentiae, a section securing goodwill, the narratio, a description of the occasion of the letter, the petitio or dispositio, a request or demand and the conclusio, a formulaic ending" (Wood, 2004, p. 235). Sur le "cadre épistolaire», voir BrancaRosoff, 2015, p. 42-43. 


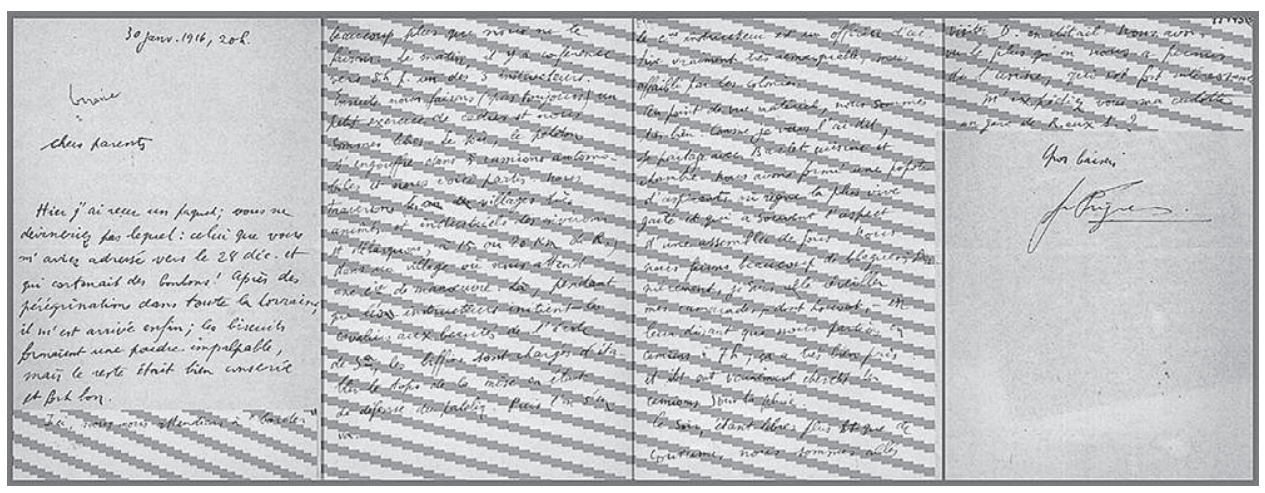

Figure 4. Part de la narration (hachures) dans une lettre d'A. Fugier (30/01/1916)

(c) Historial de la Grande Guerre (Péronne, Somme)

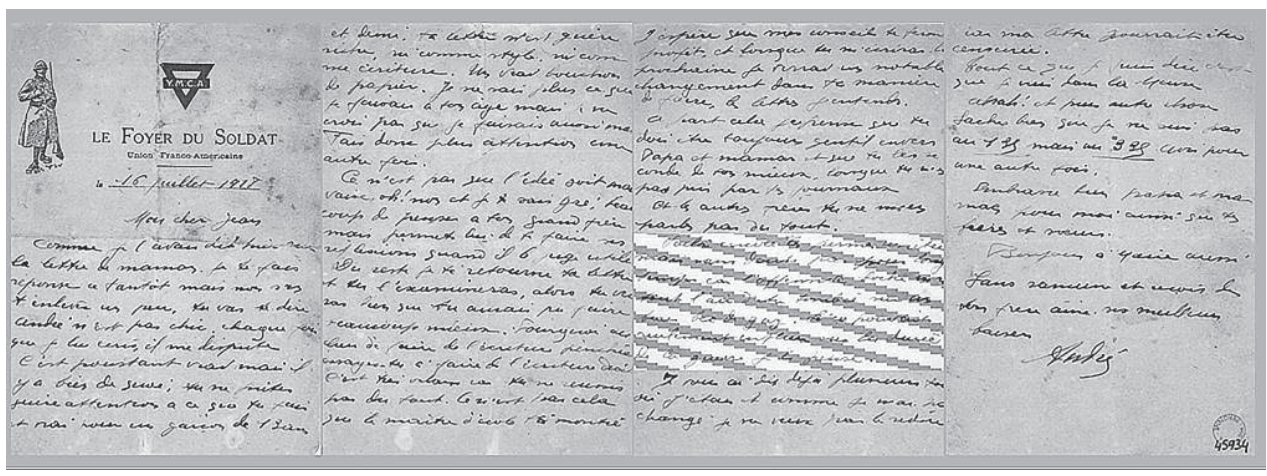

Figure 5. Part de la narration (hachures) dans une lettre de A. Boulo (16/07/1917)

(c) Historial de la Grande Guerre (Péronne, Somme)

Le cœur du texte est plutôt à tendance narrative (absence du tu et/ou du vous), tandis que la partie conclusive revêt de nouveau les caractéristiques du dialogue. C'est le poids respectif des secteurs significatifs qui varie d'une lettre à l'autre (voir à titre emblématique les figures 4 et 5). Chez A. Fugier, l'interaction est limitée aux formules d'adresse et de conclusion; chez A. Boulo, elle est maintenue presque tout au long de la lettre.

Le moindre recours au je chez A. Fugier s'expliquerait donc par un discours plus narratif que dialogal. Ce résultat, à éprouver en étudiant d'autres catégories d'observables (recours à l'implicite et à l'explicite, spontanéité de l'écrit, etc.), doit aussi être nuancé par la prise en compte de la situation d'énonciation, notamment des destinataires des lettres. Un scripteur qui écrit à ses parents, auxquels il veut montrer son émancipation et sa maturité, a certainement moins à cœur de faire exister le rapprochement je-tu/je-vous qu'un mari qui écrit à sa bien-aimée. 


\section{Image de soi et construction identitaire sociale : comment le scripteur marque-t-il ses appartenances dans la langue?}

La tendance à l'effacement du je chez A. Fugier pourrait aussi venir du large investissement du nous soldats (574 manifestations du nous exclusif). Cet investissement peut provenir d'une assimilation au groupe des soldats plus marquée chez le jeune aspirant (fils d'officier et qui envisageait une carrière militaire) que chez ses frères d'armes. Il peut aussi s'expliquer par des habitudes d'écriture prises dans l'exercice scolaire secondaire où il fallait savoir marquer une distanciation à travers le nous d'auteur pour laisser place à la rationalité et élaborer un texte valant par sa construction, ses arguments, son érudition plutôt que par sa charge émotive. À l'école primaire, par contre, c'était sur le développement de l'expression personnelle qu'on insistait : "dans le système scolaire de la Troisième République, clivé en deux ordres, primaire et secondaire, les écritures de soi apparaissent comme emblématiques de l'école primaire » (Bishop, 2010, p. 10). Il s'agissait d'y apprendre à exprimer son point de vue, à dire son ressenti.

Il faut que l'enfant, au sortir de l'école, soit en mesure d'exprimer par écrit à la première personne des sentiments ou des impressions. (Chervel, 2006, p.706)

Je ne prétends pas que l'école primaire forme des écrivains et des poètes. Son but est de préparer des hommes libres, [...] qui aient la conscience d'une vie et d'une activité propres, si modestes soient-elles, dans le travail universel. Et c'est pourquoi j'ai conseillé : faites de la rédaction française une excitatrice de la personnalité; traduisez: de l'activité et de la sincérité enfantines. (Blanguernon, 1913, p. 63)

L'investissement du nous exclusif / nous d'auteur permettrait au soldat à la fois de s'inclure

dans une communauté et [de crédibiliser] sa parole, non parce qu'elle est singulière, mais parce qu'elle est conforme à celle qu'auraient énoncée les pairs dans des circonstances analogues, et lui donnent ainsi une certaine objectivité. (Tutin, 2010, p.40)

Le nous soldat permet à A. Fugier de s'inclure dans la communauté des poilus et de crédibiliser sa parole, qu'il présente du coup comme conforme à celle des autres soldats.

Un autre critère de différenciation entre écrits primaires et écrits secondaires se trouverait donc dans la façon de dire je: moi ou nous. Chez A. Fugier, la singularité du je serait délibérément gommée au profit du collectifio. Ceci n'empêche cependant pas l'aspirant de modifier son ethos personnel : «si le je

10. Ce phénomène de fusion du locuteur dans le groupe des soldats a déjà été observé par Ruth Amossy (2010, p.172) dans son étude du carnet de route de Maurice Duwez. 
se range dans le nous, il n'en projette pas moins un ethos individuel à travers une apparente fusion dans la collectivité» (Tutin, 2010, p.177).

(10) Papa a bien tort de se déranger pour tous ces enterrements; chez nous, sur la ligne, ça se fait beaucoup plus simplement. (Fonds Fugier, lettre du 04/03/1917)

Dans cet extrait, la prise de distance d'A. Fugier par rapport aux valeurs de sa famille se fait en manifestant ostentatoirement l'appartenance au groupe des soldats, groupe dont les représentations collectives s'élaborent en réaction à celles d'avant-guerre. A. Fugier, en privilégiant son appartenance militaire ${ }^{11}$ ("chez nous »), montre qu'il se retrouve plus dans les valeurs des combattants sur le front (ici, rites funèbres simplifiés) que dans celles de ses parents et de la société civile (rites funèbres traditionnels). A. Fugier se sert de l'apparente fusion dans la collectivité des soldats pour montrer qu'il est capable d'opinions différentes de celles de ses parents : l'investissement de l'ethos collectif de soldat modifie l'image individuelle d'A. Fugier. Le fils respectueux des conventions sociales et de l'autorité paternelle fait place à un homme mature, soldat rodé à la banalité de la mort. Les changements dans l'ethos personnel préalable se font sous couvert d'ethos discursif collectif.

Chez A. Fugier, le retravail de l'ethos préalable se fait tantôt de façon manifeste comme dans l'extrait (10), tantôt de façon plus subtile. Tout se passe comme si l'aspirant voulait montrer avec ostentation, d'un côté, son appartenance au corps militaire et sa parfaite connaissance de ce milieu, de l'autre, ses capacités à la prise de distance vis-à-vis de la pression de conformité du groupe des soldats.

La part du nous par rapport au je, beaucoup plus marquée que chez les autres scripteurs, marque un fort degré d'incorporation au groupe des soldats. La finesse de la catégorisation, qui distingue hiérarchie, unité, origine et état (tableau 3$)^{12}$, montre, quant à elle, que le milieu militaire est familier au fils d'officier qu'est A. Fugier.

11. «Chacun de nous a plusieurs groupes d'appartenance [...]. Par son appartenance à un groupe, l'individu participe à une vie collective qui l'imprègne de certaines valeurs, de normes (règles de conduite), de stéréotypes [...] et subit une pression de conformité à laquelle il se plie habituellement sans problème. Un conflit surgit si l'individu prend pourvaleurs, normes, critères de jugement et de conduite, les idées et les idéaux d'un autre groupe (appelé alors groupe de référence) représentant ses aspirations personnelles ou morales » (Mucchielli, Mucchielli-Bourcier, 1969, sous l'entrée «Appartenance»).

12. Chez A. Boulo, H. Rivière et B. Lapouge, les classifications sont moins techniques et plus affectives (par exemple, je + les copains, je + un camarade ou encore je + les soldats paysans). 


\begin{tabular}{|c|c|c|}
\hline Catégorie du référent & Référence & Valeurs de nous \\
\hline Cercle familial & je + destinataires & nous $_{1}$ \\
\hline \multicolumn{3}{|l|}{ Corps militaire } \\
\hline distinction de hiérarchie & $\begin{array}{l}\text { je }+ \text { aspirant(s) je + sous-officiers je } \\
+ \text { officiers }\end{array}$ & nous $_{2}$ \\
\hline distinction d'unité & $\begin{array}{l}\text { je }+ \text { division je }+ \text { régiment je }+ \\
\text { bataillon je + compagnie je + section }\end{array}$ & nous $_{3}$ \\
\hline distinction de nationalité & je + les soldats français & nous $_{4}$ \\
\hline distinction géographique & je + un sous-officier grenoblois & nous $_{5}$ \\
\hline distinction d'état & je + soldats & nous $_{6}$ \\
\hline Emplois ambigus & & nous $_{7}$ \\
\hline
\end{tabular}

Tableau 3. L'emploi du nous dans la correspondance d'A. Fugier

Elle met aussi paradoxalement en relief les emplois indéterminés :

(11) Nous [les aspirants et/ou les soldats de la $19^{\text {e }}$ division] voyageons beaucoup, mais nous [les aspirants et/ou les soldats de la 19e division] voyons peu de pays, car nous [les aspirants et/ou les soldats de la $19^{\mathrm{e}}$ division] ne faisons que tourner en rond. C'est ainsi que nous [les aspirants et/ou les soldats de la $19^{\mathrm{e}}$ division] croiyons avoir quitté les environs de Luneville pour ceux de Nancy et maintenant me voici revenu dans ce bourg où j'ai suivi les cours du peloton de chefs de section ! Comme je vous le disais en une courte carte, nous [les aspirants] avons rejoint le 314 à M. et nous [les aspirants] nous y sommes présentés au colonel qui nous [les aspirants] a très bien reçus. Comme j'étais affecté au $5^{\text {è }}$ Bon qui ne cantonnait pas à M., j'ai été mis en subsistance à la $23^{e ̀ ~ o u ̀ ~ j ' a i ~ p a s s e ́ ~ l a ~ j o u r n e ́ e, ~ m a n g e a n t ~ a ̀ ~ l a ~}$ popote des officiers et avec laquelle j'ai fait le lendemain une étape qui nous [les soldats de la $23^{\mathrm{e}}$ division] a conduits près de Nancy. J'ai pu alors rejoindre la $19^{\mathrm{e}}$.

Le lendemain nous [les soldats de la $19^{\mathrm{e}}$ division] nous sommes appuyé $28 \mathrm{~km}$ et nous [les soldats de la $19^{\mathrm{e}}$ division] voici donc à R. aux S. (Fonds Fugier, lettre du $12 / 02 / 1916)$

Dans l'extrait (11), la référence des nous, pour être retrouvée, exige une lecture attentive et une bonne connaissance du cotexte large. Les nous du premier paragraphe peuvent référer soit aux aspirants - si l'on se fie au contenu de la

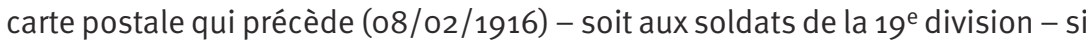
l'on se fie au contenu du second paragraphe J'ai pu alors rejoindre la $19^{\grave{e} .}$

L'économie procurée par les pronoms se paie d'une perte de clarté. Mais cela peut aussi représenter un avantage pour le locuteur puisque l'ambiguïté du nous permet de laisser les sujets concernés par l'énonciation dans une zone d'ombre relative. (Labbé, 1998, p. 6)

Le nous semble être utilisé dans la correspondance d'A. Fugier comme un je qui se revendique membre du groupe des soldats, la précision, si précision il y a, est en boni. 
(12) Quant aux hommes, la flemme du haut Commandement leur ayant fait perdre ici 5 mois qui auraient été si utiles à leur instruction, ils sont beaucoup moins dégourdis qu'en quittant le dépôt et je crois que les gradés auront fort à s'agiter pendant le voyage s'ils veulent que les hommes soient installés à peu près convenablement. Ça ne sera pas comme au 222 où on se contentait d'indiquer de loin à la Son son cantonnement et où on allait droit à la popote. (Fonds Fugier, lettre du 16/04/1916)

Dans l'extrait (12), A. Fugierva jusqu'à recourir au on pour marquer une distanciation telle qu'on ne sait s'il se catégorise parmi les gradés ou pas. Cette indétermination catégorielle lui permet de poser un regard critique sur ce dont il est spectateur. Le recours limité au je - évinçant la subjectivité du discours et les tournures impersonnelles - cachant une critique acerbe du haut commandement - jouent le même rôle et posent $A$. Fugier en témoin objectif des évènements auxquels il assiste.

(13) voici qu'on laisse des sergents avec 10, 15 hommes pour établir des feuillées, - que chaque homme doit rapporter chaque jour 4 pierres plates pour faire un pavage devant le bureau du Ct, - que les inspections de cantonnement sont quotidiennes. ah, que c'est intéressant d'être fonctionnaire adjt dans ces conditions là! (Fonds Fugier, lettre du 29/04/1916)

Par le brouillage de la référence que constituent emplois ambigus, recours limité au je et tournures impersonnelles, l'aspirant se fond dans le groupe des combattants, tout en conservant une objectivité distante et une attitude critique. L'investissement de l'identité de soldat n'empêche pas A. Fugier d'être circonspect vis-à-vis du militaire. Son rang, entre gradés et hommes de troupe, lui permet, en tant qu'aspirant, de se classer tantôt parmi les officiers (14), tantôt parmi les soldats (15):

(14) Puis je suis présenté aux ssoff. [...]. Ils me conduisent à leur bâtiment, très solidmt bâti en bois, à $50 \mathrm{~cm}$ du sol, avec fenêtres à glissières en papier huilé; on dort sur des bas flancs recouverts de paille et l'on mange dans une salle à part, dans des assiettes! Nous sommes aussi bien que des artilleurs! (Fonds Fugier, lettre du 10/10/1915)

(15) Ici, on va nous préparer à la guerre de mouvement et à l'exploitation du succès. Il s'agit d'adapter l'ancien règlement au nouvel armement. Ce n'est pas chose commode pour nos officiers. Ayant péniblement réussi à comprendre quelque chose à la guerre de tranchées, ils veulent appliquer cette science qui a été si dure à acquérir à la guerre de mouvement. De sorte qu'hier mon Mollard, en terrain découvert, a amené la section en 4 vagues se suivant à 15 pas jusqu'à 30 mètres d'une mitrailleuse ennemie qu'il a alors fait attaquer à la grenade... (Fonds Fugier, lettre du 21/02/1917)

L'instabilité catégorielle qui en découle lui donne la possibilité de faire preuve d'esprit critique sans remettre en cause une appartenance militaire assumée dans tout l'échange épistolier. 
Le jeu sur la précision de la référence - claire lorsqu'il s'agit d'afficher son appartenance et son adhésion aux valeurs d'un groupe ou d'un sous-groupe militaire spécifique (16), brouillée quand, tout en assumant son appartenance militaire, il veut marquer sa distanciation par rapport à l'idéologie du groupe (17) - lui permet d'endosser l'identité double de soldat-citoyen, c'està-dire d'homme qui, quoique soumis à l'institution militaire, ne renonce pas entièrement à ses droits de citoyen que sont la liberté de pensée et la liberté d'expression ${ }^{13}$. S'il se met au service de l'armée, le soldat-citoyen se laisse la possibilité d'une distance critique vis-à-vis du militaire.

(16) Nous achetons qq bouquins à M. et chacun a sa spécialité : moi je fais de la philosophie, Privat de l'allemand, Beaufort de la chimie, Barlet de l'anglais. C'est ce qui s'appelle des aspirants sérieux! (Fonds Fugier, lettre du 02/04/1916)

(17) La grande nouveauté de la semaine a été la constitution des spécialités et les exercices de détail sont détrônés par l'exercice des spécialistes. Ç'est très curieux; on exerce les mitrailleurs sans avoir de mitrailleuses, les signaleurs sans fanions, les grenadiers sans grenades et les sapeurs sans outils. (Fonds Fugier, lettre du 21/02/1917)

Le nous de l'extrait (16) et le on de l'extrait (17) ont le même référent, soit les aspirants. Le choix des pronoms, modulant l'implication subjective du locuteur, marque une assimilation plus ou moins assumée au groupe.

Chez A. Boulo, au contraire, c'est à l'aide d'une référence anaphorique ou déictique explicite et précise que s'opère la modulation de l'ethos préalable dans l'ethos discursif (du soldat patriote au soldat-citoyen). Dans la première partie de l'extrait (18), A. Boulo utilise nous pour désigner les Français en tant que groupe unifié; il «altérise »l'ennemi par le pronom ils et par le terme injurieux salauds.

(18) Voilà encore quelque chose qui ne va pas abréger la fin de la guerre, c'est la secousse que les Italiens viennent de se faire passer. On a intercepté un radio boche qui annonce 80000 prisonniers 600 canons et un matériel considérable. La IIIeme Armée Italienne aurait été enveloppée pendant sa retraite. La nouvelle est confirmée par le journal de Genève d'hier. Quelle tuile. Il y a plusieurs divisions françaises de parties pour là-bas. On parle même que la nôtre irait aussi, mais çà n'est que des bruits qui courrent, mais çà demande confirmation C'est la réplique a notre avance de l'Aisne et ils font aussi bien sinon mieux. Les salauds. Je crois bien qu'ils finiront par nous avoir. (Fonds Boulo, lettre du 30/10/1917)

Cet usage référentiel est conforme à l'usage que l'on attend d'un poilu patriote. Dans la suite du texte, A. Boulo continue à utiliser l'opposition nous/ ils, mais les référents changent :

(19) Avec ce qui se passe en ce moment dans les gouvernements Français et Italiens çà sent mauvais. Surtout en France ou ils se conduisent comme des mala-

13. "By definition, the citizen-soldier will not entirely relinquish the rights of the citizen " (Smith, 1994, p. 11). 
droits avec les perquisitions de l'Action Française, ils vont se couvrir de ridicule car ils montrent que Daudet avait raison et qu'ils en ont peur. Quelle bande de saligauds ils ne voient que le pognon, c'est leur Dieu a eux autres, ils ne se foutent pas mal des pauvres poilus qui se font casser la figure que «tandis〉 que'ils s'enrichissent avec l'argent Boches et celui des Français. Quón les flanque a la porte mais çà sera toujours pareil, que ce soit les uns ou les autres c'est canaille et Compagnie. Daudet fait campagne pour la royauté mais ce n'est pas la [x] forme du gouvernement qu'il faut critiquer mais les dirigeants. (Fonds Boulo, lettre du 30/10/1917)

Les référents changent, mais il n'y a pas d'ambiguïté : le nous, implicite, désigne désormais en creux les poilus, ils désigne les dirigeants politiques. A. Boulo va jusqu'à utiliser le synonyme de l'injure qu'il avait utilisée pour les Allemands afin de s'en prendre aux représentants du gouvernement français : quelle bande de saligauds. Il y a là un glissement : l'antagonisme de condition l'emporte sur l'antagonisme de nation. Les politiciens sont accusés de s'enrichir «avec l'argent Boches et celui des Français » au détriment des poilus au sens large. A. Boulo remet en question la soumission à l'égard du pouvoir ${ }^{14}$ ("que ce soit les uns ou les autres c'est canaille et Compagnie») et il met en avant la solidarité de condition de «poilus qui se font casser la figure ». À l'image du soldat patriote protecteur de la mère patrie et respectueux de l'ordre établi que le début de la lettre fait émerger, A. Boulo substitue une image de soldat-citoyen.

Aussi bien A. Boulo qu'A. Fugier font émerger une identité de soldat-citoyen :

soldat, et donc soumis à l'institution militaire, pris dans l'événement guerrier dont il [est] acteur, et citoyen maintenant sous les armes [son] appartenance à un corps politique qui [lui] enjoint d'obéir à une autorité dont il [est] en même temps la source, en vertu de la souveraineté populaire (Loez, 2005, sous l'entrée «Soldat-citoyen »)

C'est à ce titre qu'ils sont habilités à juger des autorités établies. Seules les modalités changent : l'un le fait par référence claire, l'autre par référence brouillée.

Le passé scolaire des scripteurs se ressent dans leurs façons d'user des pronoms personnels. La référence personnelle des échanges de B. Lapouge, A. Boulo et $\mathrm{H}$. Rivière se fait simple dans une optique de clarification du message. Dans ces textes massivement singuliers qui entendent projeter une image individuelle explicite, l'appartenance de condition de l'individu se manifeste par ses façons de dire (style primaire). Autrement dit, ces textes projettent dans l'ethos dit un ethos personnel, alors que l'appartenance sociale (l'ethos collectif) se manifeste plus clairement dans l'ethos montré. Au contraire, dans le texte d'A. Fugier, qui projette plus massivement une image de groupe (celui des soldats) - un ethos collectif dans l'ethos dit - la singularité de l'individu (son ethos personnel) se manifeste en dehors des contenus du discours (et donc dans l'ethos montré) par une stylisation, ici un brouillage de la référence

14. Sur la figure du « soldat qui se conduit bien » (Jeannel, 1884, p. 319) et qui « reste à la place que le destin lui a assignée », voir Maingueneau, 1979, p.161-166. 
pronominale permettant une implication subjective plus ou moins marquée du locuteur dans son discours.

L'image de soi produite dans les lettres de poilus est le résultat de tensions entre ethos préalable et ethos discursif, entre ethos dit et ethos montré, entre ethos personnel et ethos collectif. En jouant de ces différents ethos, les scripteurs, quel que soit leur passé scolaire, élaborent et modulent une image tenant compte des représentations de l'interlocuteur et des visées de leur discours. L'écriture de ces poilus montre que l'ascendant des codes n'empêche pas la liberté d'expression.

Les gens ne sont pas sans défense contre «le pouvoir des mots», sans doute d'ailleurs ont-ils des raisons de craindre davantage le pouvoir des "gouvernants». (Branca-Rosoff, 1990, p.34)

\section{Corpus}

Boulo André, 1917-1919, Correspondance, Fonds Boulo, Historial de la Grande Guerre (Péronne).

FUGIER André, 1915-1918, Correspondance, journal de guerre, carnet de campagne, Fonds Fugier, Historial de la Grande Guerre (Péronne).

LAPouge Baptiste, 1908-1919, Correspondance, Fonds Lapouge, Centre d'études Edmond Michelet (Brive).

RIVIÈRE Henri, 1914-1916, Correspondance, Fonds Rivière, Historial de la Grande Guerre (Péronne).

\section{Références}

Amossy Ruth, 2010, La présentation de soi : ethos et identité verbale, Paris, PUF.

BALIBAR Renée, 1999, "L'école de 1880 le français national : républicain, scolaire, grammatical, primaire», dans G. Antoine et R. Martin éd., Histoire de la langue française, 1880-1914, Paris, CNRS Éditions, https://books.openedition.org/editionscnrs/9270 (consulté le 25 juin 2019).

- 1985, L'institution du français : essai sur le colinguisme des Carolingiens à la République, Paris, PUF.

BARBUSSE Henri, 1969, Le Feu: journal d'une escouade, Paris, Flammarion.

BenVEnISTE Émile, 1966, Problèmes de linguistique générale 1, Paris, Gallimard.

BIBAuw Serge, 2007, Quand l'écrit converse. Interactions médiées et adaptation de l'écrit en messagerie instantanée, mémoire de licence, Louvain-la-Neuve, Université catholique de Louvain.

BIsHop Marie-France, 2010, Racontez vos vacances : histoire des écritures de soi à l'école primaire (1882-2002), Grenoble, Presses universitaires de Grenoble.

Blanguernon Edmond, 1918, Pourl'école vivante, Paris, Hachette.

BRANCA-Rosoff Sonia, 2015, «Rituels épistolaires ou flux verbal. Deux formes d'appro- 
priation de l'écriture», dans Entre villages et tranchées : l'écriture de Poilus ordinaires, A. Steuckart éd., Uzès, Éditions Inclinaison.

- 1990, "Conventions d'écriture dans la correspondance des soldats», Mots. Les langages du politique, $\mathrm{n}^{\circ}$ 24, p. 21-36.

ChervelAndré, 2006, Histoire de l'enseignement du français du XVII au xxe siècle, Paris, Retz.

CheVAllier Jacques, 1994, "Présentation», dans Les bonnes mœurs, CURAPP éd., Paris, PUF.

Detue Frédérik, 2013, «Le schisme littéraire des témoignages de la Grande Guerre », Vox Poetica. Lettres et sciences humaines, http://www.vox-poetica.org/t/articles/ detue2013.html (consulté le 6 juin 2014).

Eco Umberto, 2014, Construire l'ennemi et autres écrits occasionnels, Paris, Grasset.

Geffroy, Annie, 1985, "Les nous de Robespierre ou le territoire impossible», Mots, $n^{0} 10$, p. $63-90$.

GuesPIn Louis, 1985, «Nous, la langue et l'interaction », Mots, nº 10, p. 45-62.

Housıle Sylvie, 2014, Dire la guerre : le discours épistolaire des combattants français de 14-18, Limoges, Lambert-Lucas.

JAUBERT Anna, 1990, La lecture pragmatique, Paris, Hachette supérieur.

- 1987, Étude stylistique de la correspondance entre Henriette ${ }^{\star \star \star}$ et J. -J. Rousseau : la subjectivité dans le discours, Paris, Genève, Champion, Slatkine.

Jeannel Charles, 1884, Petit-Jean, Paris, Delagrave.

Kerbrat-Orecchioni Catherine, 1980, L'énonciation : de la subjectivité dans le langage, Paris, Armand Colin.

KIVINIEMI Anne-Laure, 2016, «Figure du discours et rapport de place dans les lettres de poilus», Langage \& Société, nº156, p. 97-120.

KLIPPI Carita, KIVINIEMI Anne-Laure, 2015, «L'écriture de deux frères d'armes, déshérités du français - Une caricature de la langue nationale?», dans Les variations diasystématiques et leurs interdépendances dans les langues romanes, $\mathrm{K}$. Jeppesen Kragh et J. Lindschouw éd., Strasbourg, ELiPhi, p. 175-190.

Koch Peter, Oesterreicher Wulf, 2001, «Gesprochene Sprache und geschriebene Sprache / Langage parlé et langage écrit», Lexicon der Romanistischen Linguistik (LRL), vol. I, 2, Tübingen, Max Niemeyer Verlag, p. 584-627.

LABBÉ Dominique, 1998, "Le “nous” du général de Gaulle», Quaderni di studi linguistici, n'4-5, p. 331-354.

LARIVE et FLEURY, 1911, La deuxième année de grammaire. Orthographe, rédaction, littérature, Paris, Armand Colin, $125^{\mathrm{e}}$ édition.

LOEZ André, 2005, Petitrépertoire critique des concepts de la Grande Guerre, https://www. crid1418.org/doc/textes/repertoire_critique_concepts.pdf (consulté le 25 juin 2019).

Maingueneau Dominique, 1979, Les livres d'école de la République, 1870-1914 : discours et idéologie, Paris, Le Sycomore.

Mucchielli Roger, Mucchielli-Bourcier Arlette, 1969, Lexique des sciences sociales, Paris, Entreprise moderne d'édition, Éditions sociales françaises.

Prost Alain, 1968, Histoire de l'enseignementen France, 1800-1967, Paris, Armand Colin.

RABATEL Alain, 2001, «La valeur de "on" pronom indéfini/pronom personnel dans les perceptions représentées ", L’information grammaticale, n88, p. 28-32. 
StEUCKART Agnès éd., 2015, Entre villages et tranchées : l'écriture de Poilus ordinaires, Uzès, Éditions Inclinaison.

SMITH Leonard V., 1994, Between Mutiny and Obedience: The Case of the French Fifth Infantry Division during World War I, Princeton, Princeton University Press.

Tutın Agnès, 2010, "Dans cet article, nous souhaitons montrer que... Lexique verbal et positionnement de l'auteur dans les articles en sciences humaines ", Lidil. Revue de linguistique et de didactique des langues, $n^{\circ} 41$, p. $15-40$.

VIALA François, 1896, L'enseignement moral à l'école primaire : livre de morale pratique et de lecture courante, Paris, Challamel.

VICARI Stefano, 2012, "L'éthos “poilu” dans les lettres des soldats de la Grande Guerre : hétérogénéités énonciatives et stratégies discursives ", dans $3^{e}$ Congrès mondial de linguistique française (CMLF), F. Neveu, V. Muni Toke, P. Blumenthal etal. éd., Les Ulis, EDP Sciences, https://doi.org/10.1051/shsconf/20120100149 (consulté le 15 mai 2019).

Wood Johanna L., 2004, «Text in context: A critical discourse analysis approach to Margaret Paston », Journal of Historical Pragmatics, vol. V, n² 2, p. 229-254.

\section{Résumé / Abstract / Compendio}

\section{Identité verbale et modèles d'expression dans les lettres de poilus}

Cet article se penche sur l'identité verbale construite dans les lettres de poilus : est-elle en prise directe sur les modèles d'écriture avec lesquels les épistoliers ont été familiarisés? Il importera de déterminer comment les scripteurs usent des pronoms personnels pour manifester leur présence et leurs appartenances.

Mots-clés : image de soi, identité verbale, Première Guerre mondiale, ethos montré

\section{Verbal identity and expression patterns in letters from the First World War}

This article looks at the verbal identity built in the letters of French soldiers of World War I: is it in direct contact with the writing models to which the letter-writers have been familiarized? It will be important to determine how script writers use personal pronouns to express their presence and affiliations.

Keywords: image of self, verbal identity, World War l, shown ethos

\section{Identidad verbal y modelos de expresión en las cartas de los soldados franceses de la Primera Guerra Mundial}

En este artículo se analiza la identidad verbal construida en las cartas de los soldados franceses de la Primera Guerra Mundial: ¿está directamente relacionada con los modelos de escritura con las cuáles los epistolarios se han familiarizado? Será importante determiner cómo estos redactors usaron los pronombres personales para expresar su presencia y su pertenencia.

Palabras claves: imagen de sí mismo, identidad verbal, Primera Guerra Mundial, ethos manifestado 